\title{
A Negotiation Support Tool Using Emotional Factors
}

\author{
Masahide YUASA, Yoshiaki YASUMURA, Katsumi NITTA \\ Department of Computational Intelligence and Systems Science \\ Tokyo Institute of Technology, Japan \\ E-mail: \{yuasa,yasumura,nitta\}@ntt.dis.titech.ac.jp
}

\begin{abstract}
In this paper, we introduce a new support tool for participants of negotiation through a computer network using emotional factors. The emotional factors are important in negotiation, since the participant's decision is affected by emotional factors such as their emotional disturbances, the impression of opponent's attitude and so on. In order to consider a negotiation strategy using emotional factors, we constructed a new state graph model. This model describes how participants' emotional status is affected by the opponent's proposal and the opponent's attitude. Based on this model, we made the negotiation support tool by which users exchange their proposal and their emotion by facial expression of an interface agent. This tool shows the user the proper action to take next by considering the opponent's proposal and the facial expression. We showed the availability of negotiation strategy using the state transition model by a computer simulation.
\end{abstract}

\section{Introduction}

Negotiation is a process to reach an agreement by exchanging participant's proposals each other. In recent years, several software tools to support negotiation through Internet have been developed. These tools support participants by giving advice based on the only utility value [1].

However, we often select the next proposal using not only the utility value but also various kinds of factors in practice $[2,3,4]$. For example, when we negotiate about compensation of a car accident, our decision making in negotiation is influenced by the feeling against the opponent and the emotional disturbance.

Therefore, in order to support decision making in negotiation, we made the new model including emotional factors. Based on the new model, we made the negotiation support tool which gives users the advice about the next action, considering not only the opponent's

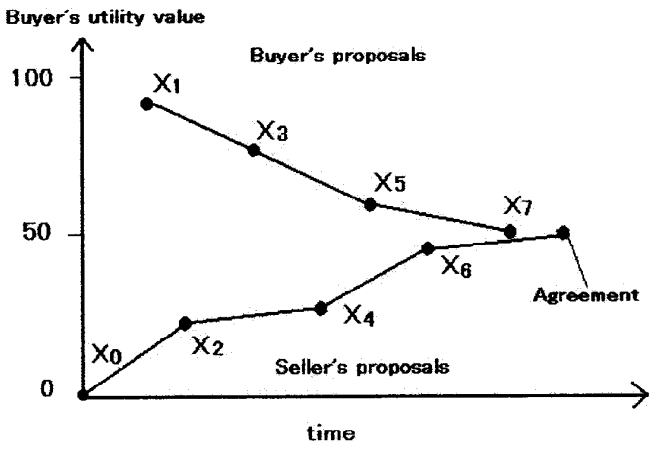

Figure 1. The utility values in negotiation

proposal but his/her emotion.

Section Two introduces the state graph model of negotiation which describes the relation among the opponent's proposal, the feeing against the opponent's attitude and the user's action. Section Three describes an overview of a negotiation support tool using this model. In Section Four, we evaluate the quality of advice of the negotiation support tool by a computer simulation. Finally, Section Five summarizes our research.

\section{Negotiation Models}

At first, we introduce the model of negotiation based on the utility value. Next, we show a new model based on Heider's A-B-X model [2] which represents human relation in social psychology.

\subsection{The Model of Negotiation Based on the Utility Value}

We will show the model of negotiation based on the utility value by a simple example $[5,6]$. In this example, two persons play the roles of buyer and seller. Figure 1 shows the typical change of the utility value of their proposals during a negotiation. Proposals are sent from one to the other in turn. $\mathrm{X} 0, \mathrm{X} 2, \mathrm{X} 4 \ldots$ mean proposals 
<smiles>[X]C1[B][Te][C]1</smiles>

(1)<smiles>[Y]1[C][B][CH]1</smiles>

(2) Unbalanced states<smiles>[X][C]1[B][C@@H]2[B][C@@H]1[CH]2</smiles>

(5)<smiles>[X]C1[B][B]C1</smiles>

(6)

Balanced states

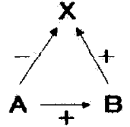

(3)

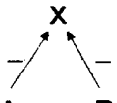

$\stackrel{+}{(7)}$

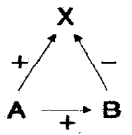

(4)

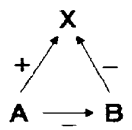

(B)
Figure 2. Balanced states and unbalanced $s$ tates
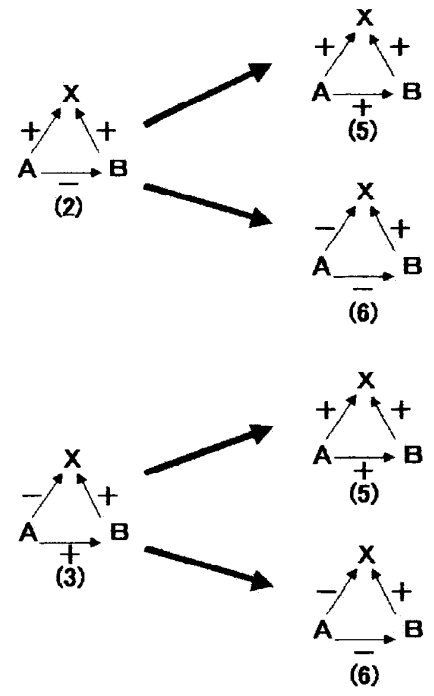

Figure 3. The changes of the state in negotiation

by the seller, and $\mathrm{X} 1, \mathrm{X} 3, \mathrm{X} 5 \ldots$ mean proposals by the buyer. In this way, the difference of their utility values decreases with time. Finally they reach an agreement when there is no difference.

Based on the model, most negotiation support tools select the next proposal. The negotiation strategy is focused on how the participants reach the agreement with the higher utility value.

\section{The Model of Negotiation Based on Hei- der's A-B-X Model}

The model of negotiation based on the utility value doesn't consider the emotional factors. In order to construct the model of negotiation including emotional fac-

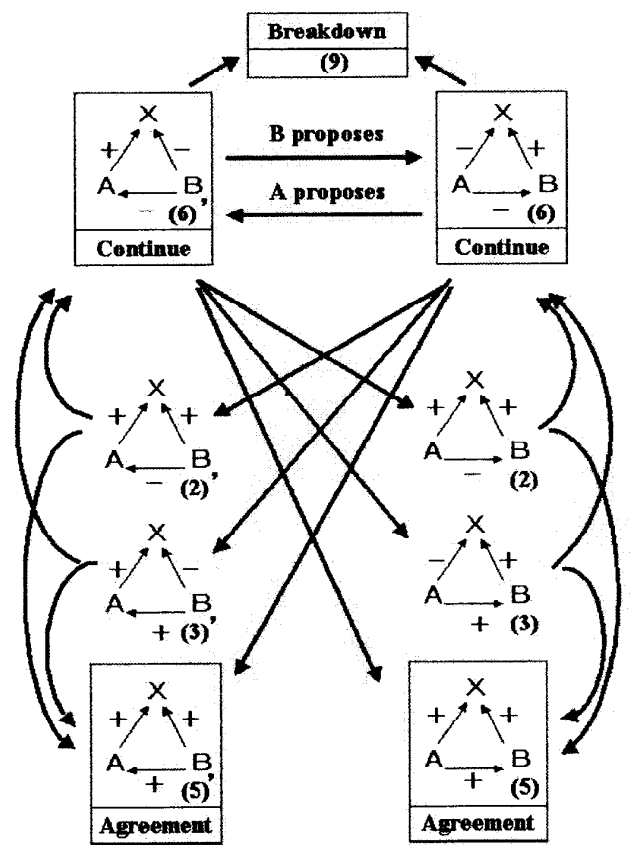

Figure 4. The state graph model using emotional factors

tors, we use the A-B-X model, which represent human relations.

A-B-X model describes a social relation among two persons, labeled $A$ and $B$, and one object, labeled X. The relation between two of these entities is indicated by the plus or minus sign. The plus sign means a person has positive feeling for the other. The minus sign means a person has negative feeling. Using this notation, social relations among two persons and one object are represented one of $8(2 \times 2 \times 2)$ status. Among them, 4 status are balanced ones and the others are unbalanced ones, as in Figure 2. And the unbalanced states tend to move to the balanced states.

In the case of negotiation, we regard A and B as participants, and $\mathrm{X}$ as a current proposal.

When B offers the proposal X, the state becomes one of (2), (3), (5) or (6). (2) means that A is satisfied with $X$ and $A$ has negative feeling to $B$. Since this state is unbalance, the state moves to the balanced state (5) or (6) in Figure 3. If A changes the feeling against B, the state moves to (5), which is an agreement state. On the other hand, if $A$ is not satisfied with $X$ because of the negative feeling against $B$, the state moves to (6). (6) means that negotiation is continued. Similarly, (3) 
means that $\mathrm{A}$ is not satisfied with $\mathrm{X}$ and $\mathrm{A}$ has positive feeling against B, after $B$ offers the proposal $X$ to $A$. And then A changes the feeling against B or to $X$, the states moves to (5) or (6).

In this way, there are three types of routes to reach an agreement after the opponent offers the proposal. The first is the route that the user is satisfied with the proposal and positive feeling for the opponent at once. The second is the route that the user has positive feeling affected by which the user is satisfied with the proposal((2)-(5)). The third is the route that the user is satisfied with the proposal affected by the user's positive feeling((3)-(5)). The third type means that the user's decision affected by the user's feeling.

Figure 4 shows that the state graph model combined these types. In Figure 4, the state (6)' means that A offers an initial proposal X. If $\mathbf{B}$ offers another proposal $X$, the state moves to this state (6). At the state (6), if B offers a new proposal $X, A$ is satisfied with that proposal and $\mathrm{A}$ has negative feeling to $\mathrm{B}$, the state moves to the state (2). And if B offers a new proposal X, A is not satisfied with that proposal and $A$ has positive feeling to $B$, the state moves to the state (3). Since the state (2) and (3) are the unbalanced state, the state moves to the balanced state (6) to continue, or the agreement state (5). Similarly, the agreement state (5)' means B is satisfied with the proposal after A offers it. The same applies to (2)' and (3)'. When A and B are not satisfied with any proposal, A or B has negative feeling strongly against the opponent or the proposal, or the time runs out, the state moves to breakdown (9).

\section{An Overview of Negotiation Support Tool using Emotional Factors}

Our system consists of a Message Control Server and Negotiation Support Tools (Figure 5).

\section{- Message Control Server}

This server is connected from the negotiation support tools to manage the participants and exchange their messages.

\section{- Negotiation Support Tool}

The support tool consists of two modules: Interface Module and Generating Advice Module.

\section{(1) Interface Module}

This module receives the opponent's proposal and the facial expression of an interface

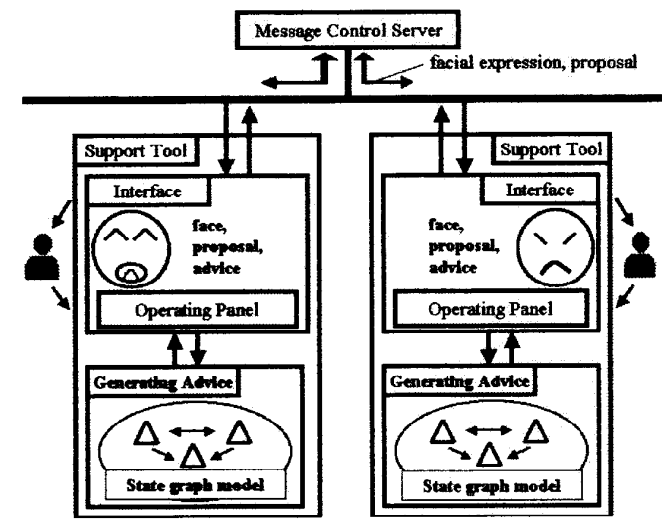

Figure 5. The architecture of the tool

agent from the opponent's tool and displays them on the screen. And it receives the advice from the Generating Advice Module and shows them. This module also displays a menu table on the screen and receives the user's proposal and facial expression.

(2) Generating Advice Module

This module receives the next proposal from Interface Module and recommends the next facial expression based on the state graph model.

\section{Learning the Strategy Using Facial Ex- pressions}

In order to generate the advice, we need to know the probability of the state transition of the state graph model. We acquired the probability by observing players actions in MONOPOLY game. Based on the probability, we made MONOPOLY agent to negotiate and we evaluate the quality of advice by a computer simulation.

\subsection{An Overview of MONOPOLY game}

The MONOPOLY is a board game played by a few players. Each player in turn puts the player's token to the place according to the dice. If the player's token stops at the place which belongs to no one, the player may purchase the place and get a card which is a deed of the title of the place. If the player's token stops at the someone's place, the player's must pay the rental fee to the owner of the card. To each card, a color (red, blue, green and so on) is attached. In order to get the much money, players must gather cards of the same color. Good players negotiate with others and gather cards of 


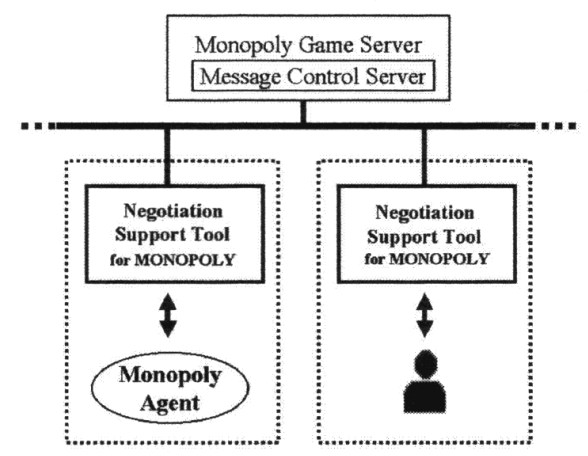

Figure 6. MONOPOLY game server and client

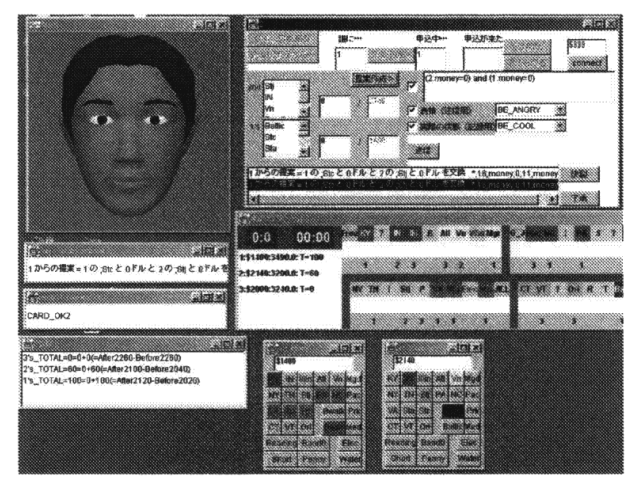

Figure 7. GUI for MONOPLOLY game on $P C$

the same color by exchanging or by purchasing cards. The end of the game is when all players are bankrupt except one.

\subsection{Learning the Probability of the S- tate Graph Model}

\subsubsection{Method}

We developed the network-based MONOPOLY game. The players participate in the game as clients by connecting to the MONOPOLY game server (Figure 6). The server manages the process of games by controlling the order of players' and their messages. Players play the game by inputting commands from the negotiation support tools. Figure 7 is the GUI for the negotiation support tool. The GUI is modified a little from the original one in order to show the board information of the MONOPOLY.

Figure 7 shows a board, cards that players have, an

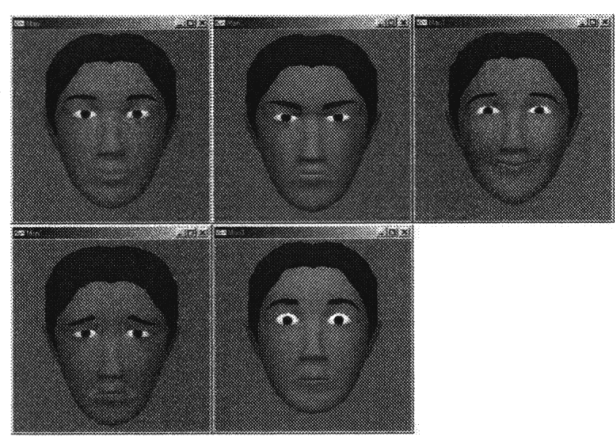

Figure 8. Facial expressions

operating panel and facial expressions on the screen. The player selects the proposal and the facial expression in the panel. Figure 8 shows facial expressions that player can select(i.e, COOL, ANGRY, HAPPY, SAD and SURPRISED [7]).

In addition, players are required to evaluate proposals on a scale of -5 to 5 as in Table 1 . This rating means how the player is satisfied with the proposal received from the opponent.

In the table, 'Ori', 'CT', 'KY', etc. are names of places which proposer wants to exchange. For example, the first row means B wants to exchange the cards, Oriental Avenue, Connecticut Avenue, with Kentucky Avenue, Atlantic Avenue. A's rating for this proposals is -3 and B's rating is 2 .

Table 1. A record of negotiation

\begin{tabular}{|c|l|c|c|c|}
\hline proposer & proposal & face & $\begin{array}{c}\text { A's } \\
\text { rating }\end{array}$ & $\begin{array}{c}\text { B's } \\
\text { rating }\end{array}$ \\
\hline B & $\begin{array}{l}\text { A= Ori, CT } \\
\text { B= KY, Atl }\end{array}$ & ANGRY & -3 & 2 \\
\hline A & $\begin{array}{l}\text { A= VA and \$100 } \\
\text { B= VT }\end{array}$ & ANGRY & 1 & -2 \\
\hline B & $\begin{array}{l}\text { A= Illi and \$300 } \\
\text { B= KY }\end{array}$ & COOL & -5 & 2 \\
\hline A & $\begin{array}{l}\text { A= VA and \$100 } \\
\text { B= VT }\end{array}$ & ANGRY & 1 & -2 \\
\hline B & $\begin{array}{l}\text { A= Illi and \$100 } \\
\text { B= VT }\end{array}$ & COOL & -2 & 0 \\
\hline A & $\begin{array}{l}\text { A= Illi and \$50 } \\
\text { B= VT }\end{array}$ & HAPPY & 0 & -1 \\
\hline B & Agreement & & & \\
\hline
\end{tabular}




\subsubsection{Result}

In the experiment, we obtained 56 cases including 292 proposals and 46 cases without facial expressions including 126 proposals. Table 2 shows that the probability of the players' actions when they received the proposal and the facial expressions from the opponents. In this table, the player's rating for the proposal is classified into 3 levels, low $(-5 \sim-4)$, middle $(-3 \sim-1)$ and high $(0 \sim 5)$.

Table 2. The player's action by the different facial expressions

- The case without facial expressions

\begin{tabular}{|r|r|r|r|}
\hline & \multicolumn{3}{|c|}{ Action } \\
\cline { 2 - 4 } Rating & Agreement & Continue & Breakdown \\
\hline high & 91 & 9 & 0 \\
middle & 21 & 77 & 12 \\
low & 0 & 73 & 27 \\
\hline
\end{tabular}

- ANGRY

\begin{tabular}{|r|r|r|r|}
\hline \multirow{2}{*}{ Rating } & \multicolumn{3}{|c|}{ Action } \\
\cline { 2 - 4 } & Agreement & Continue & Breakdown \\
\hline high & 39 & 61 & 0 \\
middle & 8 & 77 & 15 \\
low & 0 & 80 & 20 \\
\hline
\end{tabular}

- COOL

\begin{tabular}{|r|r|r|r|}
\hline \multirow{2}{*}{ Rating } & \multicolumn{3}{|c|}{ Action } \\
\cline { 2 - 4 } & Agreement & Continue & Breakdown \\
\hline high & 49 & 51 & 0 \\
middle & 4 & 94 & 2 \\
low & 0 & 96 & 4 \\
\hline
\end{tabular}

- HAPPY

\begin{tabular}{|r|r|r|r|}
\hline \multirow{2}{*}{ Rating } & \multicolumn{3}{|c|}{ Action } \\
\cline { 2 - 4 } & Agreement & Continue & Breakdown \\
\hline high & 90 & 10 & 0 \\
middle & 33 & 55 & 12 \\
low & 0 & 89 & 11 \\
\hline
\end{tabular}

- SAD

\begin{tabular}{|r|r|r|r|}
\hline \multirow{2}{*}{ Rating } & \multicolumn{3}{|c|}{ Action } \\
\cline { 2 - 4 } high & Agreement & Continue & Breakdown \\
middle & 50 & 50 & 0 \\
low & 14 & 81 & 5 \\
\hline
\end{tabular}

- SURPRISED

\begin{tabular}{|r|r|r|r|}
\hline \multirow{2}{*}{ Rating } & \multicolumn{3}{|c|}{ Action } \\
\cline { 2 - 4 } & Agreement & Continue & Breakdown \\
\hline high & 60 & 40 & 0 \\
middle & 7 & 86 & 7 \\
low & 0 & 75 & 25 \\
\hline
\end{tabular}

In the case which players don't use facial expressions, when the rating is high, the probability to reach an agreement is the highest (91\%). And when the rating is low, the probability to break down is the highest (27\%).
On the other hand, in the case of ANGRY, when the rating is high, the probability to reach an agreement is lower $(39 \%)$ than the case without facial expressions. And when the rating is middle, the probability to break down is higher (15\%) than the case without facial expressions. In addition, in the case of HAPPY, when the rating is middle, the probability to reach an agreement is the highest (33\%). And the probability to break down is the lowest $(11 \%)$.

In short, in the case that the facial expression is HAPPY, even if the player received the rating is low, the probability to break down is low.

\subsection{The Evaluation of Advice}

\subsubsection{Method}

We obtained the probability of the state transition in the state graph model. Using the probability, the negotiation support tool gives the user an advice about facial expressions which the player should use when the player shows the next proposal to the opponent. To evaluate the quality of advice, we need to play the MONOPOLY game according to the advice repeatedly and examine how much money we can get.

However, it takes much time to get the statistically meaningful data. Therefore, we conducted the evaluation of the advice by a computer simulation. We developed a MONOPOLY agent which plays the MONOPOLY game using the negotiation support tool instead of a human player.

When the MONOPOLY agent receives the opponent's proposal and the facial expression, the agent acts according to the probability (Table 2). If the agent decides to continue the negotiation, it selects the next proposal using a negotiation strategy based on the utility value, and selects the facial expression by following strategies.

- HAPPY-AGENT always selects the HAPPY face.

- ANGRY-AGENT always selects the ANGRY face.

- AVERAGE-AGENT uses five facial expressions randomly.

We observed how the agents behave and which type of agent gains the most benefit. The MONOPOLY agent selects the next proposal using the following evaluation value $U$. 


$$
U=\sum_{i} f_{i}+M
$$

$\sum_{i} f_{i}$ means amount of values of cards that the player has. Subscript $i$ means a kind of colors that the cards belong to. $M$ means money that the player has. $f_{i}$ becomes very high if the agent has all cards of the color $i$. Agents make proposals which increase the evaluation values of both the agent and the opponent in the game.

We simulated two cases. In the case (a), one HAPPYAGENT and two AVERAGE-AGENTS participated in the game. In the case (b), one ANGRY-AGENT and two AVERAGE-AGENTS participated.

Table 3. The trading to use the different facial expressions

(a)HAPPY vs AVERAGE $\times 2$

\begin{tabular}{|l|r|r|r|r|}
\hline Agent & Agreement & Continue & Breakdown & Evaluation Value \\
\hline HAPPY & 266 & 384 & 8 & 5514 \\
AVERAGE & 212 & 503 & 28 & 5179 \\
AVERAGE & 162 & 468 & 15 & 5115 \\
\hline
\end{tabular}

(b)ANGRY vs AVERAGE $\times 2$

\begin{tabular}{|l|l|l|l|l|}
\hline Agent & Agreement & Continue & Breakdown & Evaluation Value \\
\hline
\end{tabular}

\begin{tabular}{|l|l|l|l|l|}
\hline ANGRY & 194 & 625 & 31 & 5189 \\
AVERAGE & 229 & 525 & 16 & 5413 \\
AVERAGE & 217 & 522 & 17 & 5432 \\
\hline
\end{tabular}

\subsubsection{Result}

The result is shown in Table 3. In the case of (a), 120 games including 368 cases were executed. The case to reach an agreement for HAPPY-AGENT is 151 , the case to continue is 232 and the case to break down is 4 . And the average of evaluation value at the end of one game is 5431. In the case of (b), 110 games including 311 cases were executed. In the case of (a), the strategy to use HAPPY enables to increase agreement. At the case of (b), the strategy to use ANGRY enables to increasc breakdown. In addition, HAPPY-AGENT gained the most benefit (5514) in three agents. On the other hand, ANGRY-AGENT's result is the worst (5189).

In short, the results show that the amount of agreement and their benefit change by the difference of the strategy using facial expression. The player is able to become to take the advantage by selecting the suitable facial expression in negotiation.

\section{Conclusion}

We introduced the new negotiation support tool using emotional factors.
In order to consider emotional factors, we applied A-B$\mathrm{X}$ model as a model of human relation to negotiation. Based on the model, we made the negotiation support tool using the facial expressions. In order to confirm the availability of the strategy using facial expressions, we made MONOPOLY agents, which have the different strategy. By the computer simulation we confirmed that we can take the advantage in negotiation by selecting the suitable facial expression.

In the future, we will consider the history of trading and the previous facial expressions that the opponent uses. By using the additional information [8], the advice can be more suitable.

\section{References}

[1] Katsumi Nitta, Akira Yamazaki, Takashi Kitou and Yoshiaki Yasumura, "A Negotiation Support Tool using a Computer Network", Technical report of IEICE, OFS98-19, AI98-28, pp53-60, 1998, (in Japanese).

[2] Isamu Saito, The Handbook of Experimental Social Psychology 2: Interpersonal Attraction and Social Motives, Seishin Shobo, 1987, (in Japanese).

[3] Leight Thompson, Victoria Husted Medvec,Venessa Seiden, "Poker Face, Smiley Face, and Rant'n' Rave: Myths and Realities about Emotion in Negotiation", Blackwell Hankbook in Social Psychology, Vol.3, Group Processes, 2000.

[4] Max H. Bazerman, Margaret A. Neale, Negotiating Rationally, The Free Press, 1997.

[5] Gregory Kersten,Sunil Noronha, "Supporting International Negotiation with a WWW-based System", IIASA, IR-97-49, 1997.

[6] Gregory Kersten,Sunil Noronha, "Negotiation and the Web: User's Perceptions and Acceptance", IIASA, IR-98-002, 1998.

[7] Osamu Hasegawa and Katsuhiko Sakaue, "CG Tool for Constructing Anthropomorphic Interface Agents", Proc. IJCAI-97 WS (W5), ANIMATED INTERFACE AGENTS, Nagoya, Japan, 1997-8, pp.23-26.

[8] Masahide YUASA, Yoshiaki YASUMURA, Katsumi NITTA, "A Negotiation support tool using Physiological Information", IEEE SMC, Nashville, USA, October 2000, pp248-253. 